\title{
Deep Inferior Epigastric Perforator Flap (D.I.E.P) for Breast Reconstruction: Impact of Intraoperative Intrathecal Morphine on Outcome
}

\author{
M. Ben Aziz, K. Halenarova, E. Schröder, V. Kamps, M. Paesmans, \\ M. Khalife, F. Urbain, and M. Sosnowski \\ Department of Anesthesiology, Jules Bordet Institute, Boulevard de Waterloo 121, 1000 Bruxelles, \\ Belgium
}

Corresponding Author

M. Ben Aziz

mohammed.benaziz@

bordet.be

\section{Editors}

Ashok Attri

Tolga Dinç

Marco Frascio

\section{Dates}

Received 1 February 2018 Accepted 28 February 2018

Copyright $\odot 2018$ M. Ben Aziz et al. This is an open access article distributed under the Creative Commons Attribution License, which permits unrestricted use, distribution, and reproduction in any medium, provided the original work is properly cited.

\begin{abstract}
Background: Pain control after breast reconstruction with Deep Inferior Epigastric Perforator (DIEP) often requires intravenous narcotic analgesia and inpatient hospitalization. Intrathecal morphine (ITM) administration analgesia is increasing in popularity because it decreases the use of intravenous analgesic medications and offer comparable pain relief with less systemic side effects. Questions/purposes: The aim of this retrospective study was to evaluate the effect of intrathecal morphine on postoperative morbidity of breast reconstruction by Deep Inferior Epigastric Perforator flap and compare it with intravenous narcotic analgesia. Methods: 269 patients underwent immediate or delayed DIEP reconstruction after mastectomy, by the same surgeon, at Jules Bordet Institute. Patients receiving ITM analgesia (300 $\mu$ ) were matched 1:3 with patients undergoing intravenous narcotic analgesia for pain control in the same years by the same surgeon. Differences in peri- and postoperative complications across the two groups were assessed. Results: The two groups were comparable in terms of demographic characteristics and factors of morbidity. Intraoperative variables were not statistically different between the groups except for intraoperative blood loss $(P=0.0001)$, transfusion $(P=0.0001)$ and Intraoperative liquid requirement $(\mathrm{p}=0.0001)$. Intra and postoperative blood pressure were lower in ITM group $(\mathrm{p}<0.05)$. Patients in ITM group showed lower postoperative analgesia requirement $(\mathrm{P}<0,0001)$, less respiratory complications and less acute respiratory failure $(\mathrm{P}$ $=0,003$ and $\mathrm{P}=0,004$, respectively). No statistically significant differences in the length of hospital stay (LOS) were noted Conclusion: We found that intrathecal morphine analgesia was associated with less blood loss and fluid administration, better postoperative pain control, and less respiratory complications with an acceptable security profile than intravenous narcotic analgesia. Clinical relevance: This study suggests that using intrathecal morphine may result in less blood loss and blood transfusion, better postoperative pain control, and less respiratory complications.
\end{abstract}

Keywords: intrathecal morphine; general anesthesia; flap breast reconstruction, hypotension.

\section{Introduction}

In 2017, an estimated 255,180 new cases of breast cancer (including 2,470 cases in men) will be diagnosed, and 41,070 deaths (including 460 deaths in men) will occur in United States [1].

This represents a major change in morbidity and mortality specific to women [2]. 
While breast conservation techniques are available for local control of the disease for many patients, not all patients are good candidates for these techniques. Surgery is the primary modality in the management of resectable breast cancer, and when integrated with other therapies plays a significant role in controlling locally advanced or metastatic disease.

The surgical treatment of breast cancer was one of the first systemized surgical treatments during the first surgical revolution at the end of the 19th century. The scientific description of the radical mastectomy by Halstead remains an example of how oncologic treatment started off barely 120 years ago [3].

The National Institute for Health and Clinical Excellence (NICE) recommends that reconstruction should be offered to all patients with breast cancer, but the type of reconstruction to use is currently at the discretion of the surgeon and patient [4]. Methods of reconstruction include implant reconstruction and autogenous reconstruction. Autologous reconstruction techniques are either vascularized skin/muscle flaps (latissimus dorsi flap, TRAM flap), or free flaps (DIEP Flap, superior gluteal flap) and allow either the reconstruction after skin-sparing mastectomy or the plastic reconstruction after loss of the breast skin as part of a traditional mastectomy.

The deep inferior epigastric perforator (DIEP) flap, introduced by Holmstrom and Robbins [5], is a reliable and reproducible technique for autologous microsurgical breast reconstruction after mastectomy for breast cancer [6,7].

Koshima and Soeda reported the first clinical use of the lower abdominal skin and fatty tissue for breast reconstruction without sacrificing rectus muscle [8]. The use of the deep inferior epigastric artery perforator flap (DIEAP) for breast reconstruction was then popularized by Allen and Treece [9]. Since that time, the use of the DIEP flap has become the gold standard for breast reconstruction in many centers $[10,11]$.

The primary advantage of the procedure is that the consistency of the reconstructed breast is similar to the natural breast in softness and in the way the tissue drapes on the chest.

In addition, the tissue is part of the patient's body, thus it does not incite a foreign body reaction or capsular contractures that have plagued implant reconstructions [12].

Although the DIEP flap achieves long lasting satisfactory results in most patients, this long lasting procedure is not devoid of complications: potential DIEP flap complications include total or partial flap loss, infection, wound dehiscence, fat necrosis, hematoma, pulmonary embolus, blood loss and Pain and weakness at the site from which the donor tissue was taken.

In contrast to the increased knowledge on flap design, complications and long term results, little is known about the anesthetic and perioperative management, which may have an effect on the outcome of DIEP flap surgery. In this way, the proper management of postoperative pain is important in both aesthetic and reconstructive surgery. It has been shown that when pain is well-controlled, outcomes are improved in orthopedic, vascular, general, and cardiac surgery [13]. Several studies have shown decreased postoperative pain when regional anesthesia techniques are used for breast surgery. If a deep inferior epigastric perforator (DIEP) flap is performed, the use of transversus abdominis plane block (TAP) has also been shown to decrease postoperative opioid use and pain scores [14]. However, no studies have reported on the effect of intrathecal morphine on DIEP flap outcomes. Thus, the purpose of our retrospective studies was to evaluate the impact of combined intraoperative intrathecal morphine with general anaesthesia on postoperative morbidity of DIEP flap surgery. 


\section{Methods}

After the approval of the Ethics Medical Committee, we reviewed the medical records and the anesthesiology charts of 269 patients who underwent flap DIEP breast reconstruction after mastectomy, from January 2006 to June 2015 by the same experienced surgeon, at Jules Bordet Institute.

The following patient characteristics were reviewed: age, gender, weight, height, body mass index (BMI), American Society of Anesthesiologists (ASA) score, past medical history, cardiovascular risk factors including diabetes and smoking, chronic medication use including anticoagulants and non-steroidal anti-inflammatory drugs (NSAID), and alcohol use. Data from the preoperative and postoperative blood tests with hematocrit/hemoglobin $(\mathrm{Ht} / \mathrm{Hb})$ as well as the tumor characteristics and their definitive histopathological examinations were noted. Intraoperative data were also collected regarding preoperative information. We gathered manuscript anesthesiology peroperative reports or INNOVIAN peroperative recorder reports (Anesthesia information management system to create a complete, continuous, paperless record of patient's anesthetic care) depending on the availability, operative and anesthesia time, type of anesthesia with the products used and their dosages, type and quantity of fluid resuscitation, hemodynamic data and intraoperative blood loss, transfusion and diuresis. Postoperative data were also gathered in ICU postsurgical files: we noted postoperative (24 hours) blood loss, morphine and piritramid (synthetic opioid analagesic) consumption, respiratory complications, cardiac complications and infectious complications. Two groups were constituted based on the type of anesthesia received: general anesthesia alone or general anesthesia combined with intrathecal morphine.

General endotracheal anesthesia was given intravenously according to the standards used in our institute. Patients were invasively monitored, with an arterial line, a central venous line and a Core Temperature measurement. Hemodynamic monitoring included cardiac monitoring by electrocardiogram (ECG), noninvasive blood pressure (NIBP), oximeter, capnography (ETCO2: end-expiratory carbon dioxide concentration) and bispectral index (indicating the level of sedation and guiding the administration of anesthetics agents to maintain adequate hypnotic level). The patient's body temperature was kept constant by a heating blanket.

When intrathecal morphine procedure was combined to general anesthesia, the patient was in a sitting position for the puncture of intrathecal morphine. Local anesthesia was performed on the space between L4 and L5 after anatomical spotting. Lumbar puncture was performed under aseptic conditions, using a $25 \mathrm{G}$ Quincke spinal needle. ITM $(0.3 \mathrm{mg})$ was administered as a single bolus dose.

\subsection{Statistical analysis}

Statistical analysis were performed using chi square tests or Fisher exact test for categorical variables and using non parametric Mann-Whitney tests for continuous variables.

Variables were presented in term of median and interquartile range (IQR). In order to compare the two groups, were tested continuous variables for normality (Shapiro-Wilk).

All of the tests were two-sided and performed with a 5\% alpha risk. 
Table 1: Patient's baseline characteristics.

\begin{tabular}{l} 
Characteristic \\
Age \\
Median/ IQR \\
BMI \\
Median/IQR \\
Missing \\
ASA \\
I \\
II \\
Missing \\
Smoking \\
Alcohol \\
Diabetes \\
Arteriel Hypertention \\
Chronic obstructive \\
pulmonary disease (COPD) \\
Infarctus Heart Desease \\
Preop anti-aggregants \\
Preop anticoagulants \\
Nonsteroidal \\
anti-inflammatory drugs \\
(NSAIDs) \\
Preop lung disease \\
\hline
\end{tabular}

\begin{tabular}{|c|c|}
\hline Intrathecal $M+(n=176)$ & No intrathecal $M+(n=93)$ \\
\hline $52 / 44-59$ & $49 / 43-57$ \\
\hline $\begin{array}{c}24.8 / 22.5-27.3 \\
1\end{array}$ & $24.8 / 22.4-28.0$ \\
\hline $\begin{array}{c}27(16 \%) \\
147(84 \%) \\
2\end{array}$ & $\begin{array}{l}19(20 \%) \\
74(80 \%)\end{array}$ \\
\hline $22(13 \%)$ & $17(18 \%)$ \\
\hline $24(14 \%)$ & $15(16 \%)$ \\
\hline \multicolumn{2}{|c|}{ Not reported 3 patients only with diabetes } \\
\hline $43(24 \%)$ & $14(15 \%)$ \\
\hline \multicolumn{2}{|c|}{ Not reported only 1 patient with COPD } \\
\hline \multicolumn{2}{|c|}{ No patient } \\
\hline \multicolumn{2}{|c|}{ Not reported no patient received those agents } \\
\hline \multicolumn{2}{|c|}{ Not reported no patient received those agents } \\
\hline \multicolumn{2}{|c|}{ Not reported only 2 patients received those agents } \\
\hline $17(10 \%)$ & $9(10 \%)$ \\
\hline
\end{tabular}

\begin{tabular}{|c|}
\hline $\mathrm{p}$ \\
\hline 0.09 \\
\hline 0.92 \\
\hline 0.31 \\
\hline 0.22 \\
\hline 0.58 \\
\hline 0.07 \\
\hline
\end{tabular}

\section{Results}

Demographics and preoperative characteristics of all included patients $(n=269)$ are reported in Table 1. The two groups did not differ on those characteristics.

In regard with intraoperative variables, statistical analysis showed a significant principal effect of group on blood loss quantity $(\mathrm{p}<0.0001)$, red cell transfusion $(\mathrm{p}<0.0001)$, intraoperative hypotension $(\mathrm{p}=0.04)$, opioids given $(\mathrm{p}<0.05)$, quantity of crystalloids given $(\mathrm{p}<$ $0.0001)$ and surgery duration $(\mathrm{p}=0.02)$ as reported in Table 2 and Figure 1 and 2. No difference was observed regarding the anesthesia time and intraoperative quantity of colloids given (all p's $>0.05)$.

As for postoperative complications (Table 3), chi square analysis revealed that intrathecal morphine group had significantly less respiratory complication $(p=0.003)$ and acute respiratory failure $(\mathrm{p}=0.004)$ leading to non-invasive ventilation according to BIPAP ventilation mode ( $\mathrm{p}$ $<0.0001)$.

Intrathecal morphine group had a higher rate of patients presenting postoperative hypotension $(p=0.002)$. Bonferroni post-hocs tests revealed that ITM group significantly differed from the general anesthesia alone group in terms of postoperative piritramide consumption $(\mathrm{p}<0.0001)$

The intrathecal morphine administration was associated with neither the length of hospital stay nor the occurrence of flap complications (Table 3).

There were no other statistically significant differences between groups regarding neurologic complications as observed in Table 3. 
Table 2: Intraoperative data.

Characteristics

Anesthesia duration:Median / IQR

Surgery duration :Median/ IQR

Cristalloids :Median/ IQR

Colloids :Median/ IQR

Voluven :Median/ IQR

Gelofusine: $\mathrm{n}(\%)$

PerOp Blood loss: Median/ IQR

Red cells transfusion: $\mathrm{n}(\%)$

Arterial Hypotension: n(\%)

Ketamine: $\mathrm{n}(\%)$

Catepressan: $\mathrm{n}(\%)$

Remifentanil Median/IQR

Sufentanil: $\mathrm{n}(\%)$

Piritramide: $\mathrm{n}(\%)$

Morphine: $\mathrm{n}(\%)$

Dobutamine: $\mathrm{n}(\%)$

Corticosteroids: $\mathrm{n}(\%)$

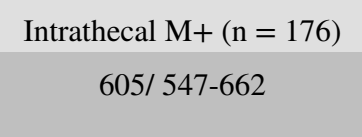

$533 / 475-590$

4000/ 3000-5000

$1000 / 500-1500$

1000/ 500-1500

$30(17 \%)$

200/ 100-300

$7(4 \%)$

$89(51 \%)$

$8(5 \%)$

$7(4 \%)$

7.2/ 5.3-10.0

0

$27(16 \%)$

$53(30 \%)$

$8(5 \%)$

$143(81 \%)$
No intrathecal $M+(n=93)$ 619/562-706

562/ 504-634

5000/ 4000-6000

1450/ 500-1500

1000/ 500-1500

19 (21\%)

300/ 125-500

22 (24\%)

$35(38 \%)$

17 (18\%)

$7(8 \%)$

7.6/ 3.6-10.0

$23(25 \%)$

$56(61 \%)$

$2(2 \%)$

$21(23 \%)$

$65(70 \%)$

\begin{tabular}{c}
$\mathrm{p}$ \\
0.22 \\
0.02 \\
$<0.0001$ \\
0.06 \\
0.23 \\
0.48 \\
$<0.0001$ \\
$<0.0001$ \\
0.04 \\
$<0.001$ \\
\hline 0.20 \\
\hline 0.25 \\
$<0.0001$ \\
$<0.0001$ \\
$<0.0001$ \\
$<0.0001$ \\
0.03 \\
\end{tabular}

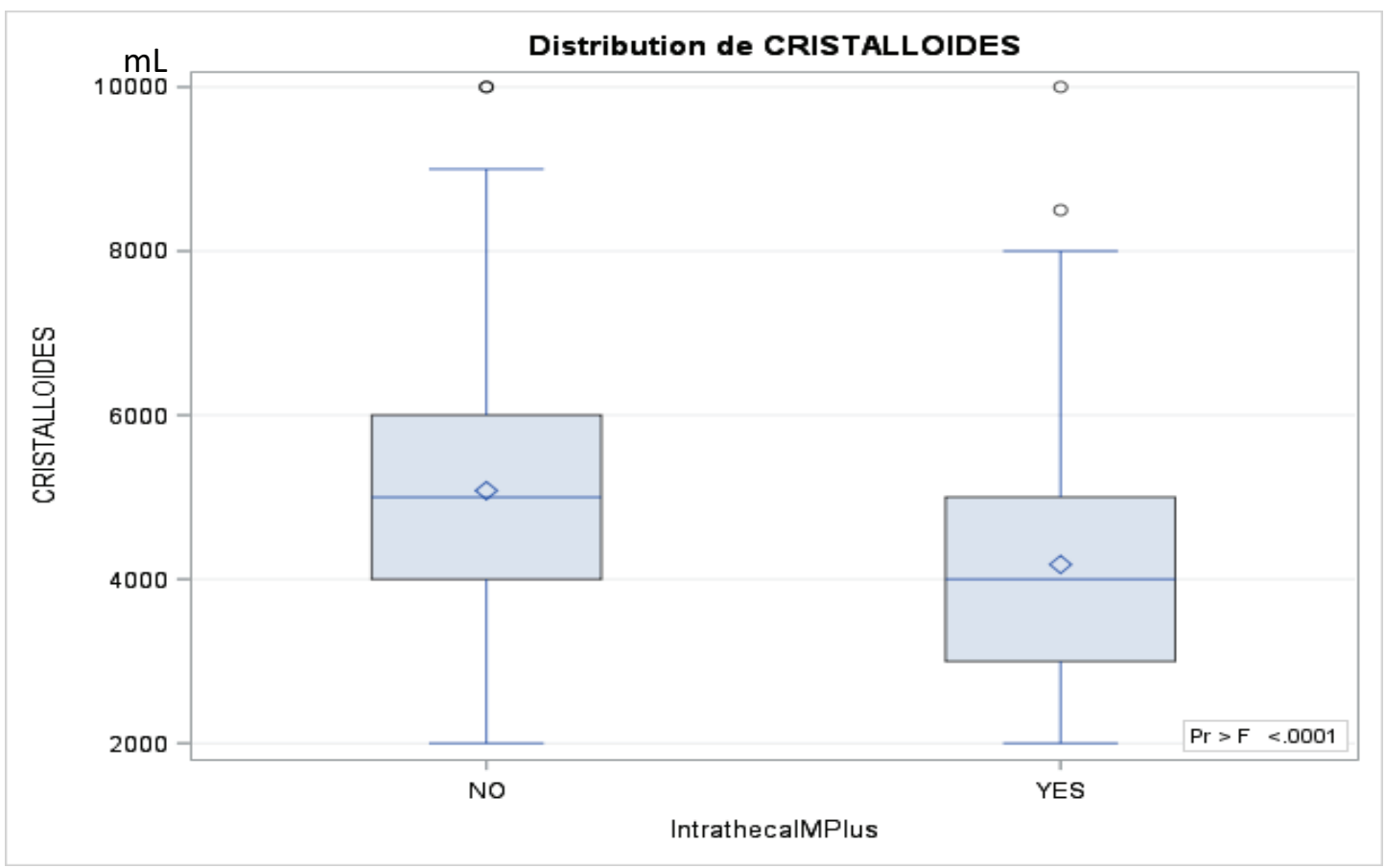

Figure 1: Intrathecal morphine is associated with less fluid administration. 


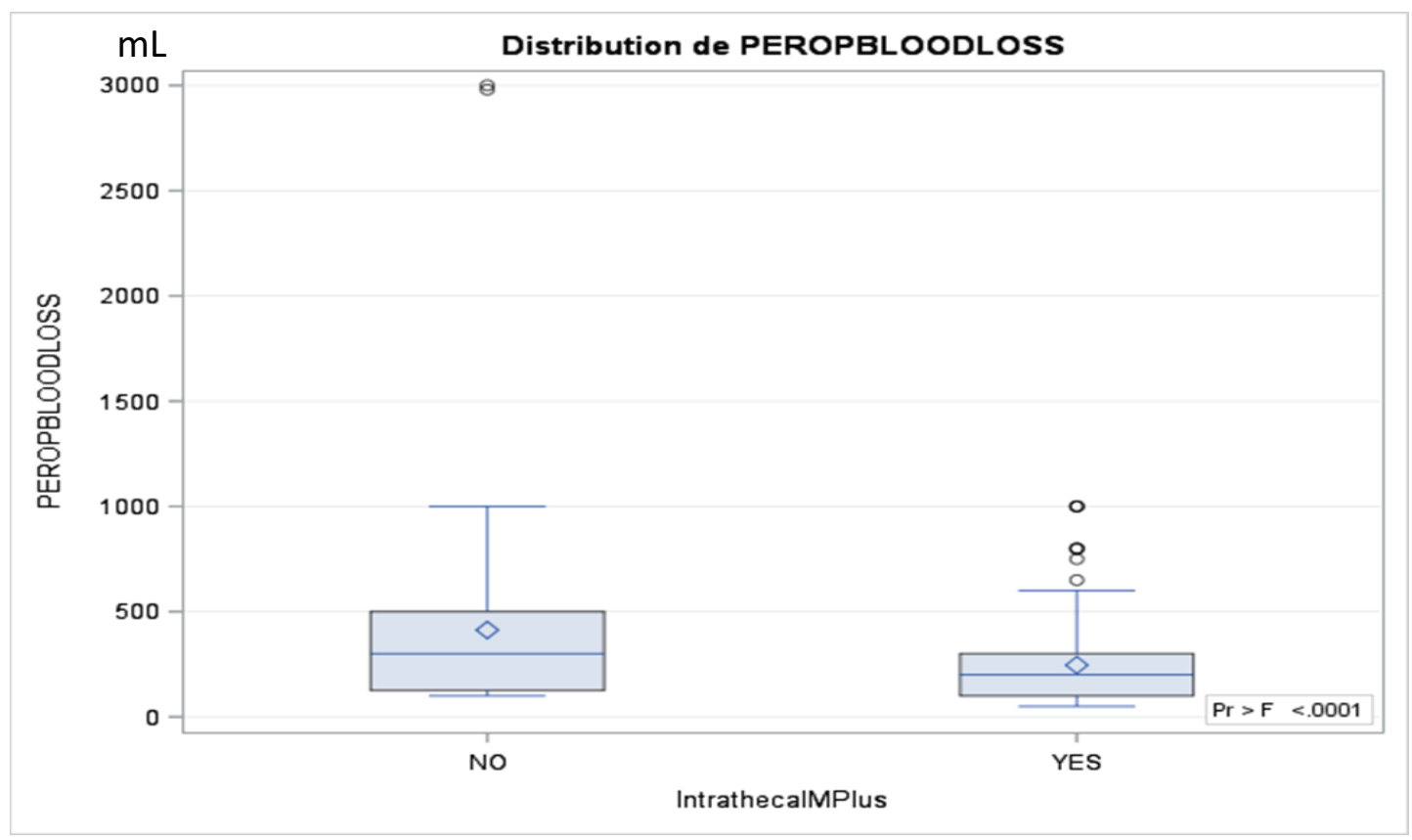

Figure 2: Intrathecal morphine is associated with less intraoperative blood loss.

\section{Discussion}

Breast reconstruction using autologous tissue has been shown to be a safe and reliable operation throughout the literature [15-17]. Moreover, DIEP flap breast reconstruction population represents a uniform group of patients amenable to specific evaluation of postoperative pain [18]. The proper management of postoperative pain is important in both aesthetic and reconstructive surgery. It has been shown that when pain is well-controlled, outcomes are improved in orthopedic, vascular, general, and cardiac surgery [19].

The acute pain literature describes a population of patients that have a poor response to narcotic use in both cancer and postsurgical care. These patients represent $25 \%$ to $40 \%$ of the population and have been previously described as "nonresponders." [20, 21]

Stamer et al [20] were the first to identify a subgroup of nonresponders who had higher pain scores and required more analgesic consumption than their "normal" counterparts. Bar Meir et al [18] found a distinct non responder subgroup of patients who had elevated pain scores despite higher morphine consumption after flap DIEP reconstruction. This group was characterized by a significantly higher VAS (visual analog score) on the first postoperative day, a greater total intravenous morphine consumption, and a longer hospital stay.

In our institution, the postoperative pain regimen for patients undergoing DIEP flap breast reconstruction is standardized using intrathecal morphine intraoperatively followed by conversion to intravenous narcotics postoperatively. Intrathecal opioid administration is an attractive analgesic technique since the opioid is injected directly into the cerebrospinal fluid, close to the structures of the central nervous system where the opioid acts.

The first clinical study testing intrathecal morphine in this context was published in 1979 [22]. Since then, this analgesic method has been the subject of a large number of trials and reviews [23-25], illustrating an ongoing interest in the technique. 
Table 3: Postoperative complications.

\begin{tabular}{l} 
Characteristics \\
Blood loss Day 1 : Median/ IQR \\
Piritramide Day 1: $\mathrm{n}(\%)$ \\
Morphine Day 1: $\mathrm{n}(\%)$ \\
Hypotension Day 1: $\mathrm{n}(\%)$ \\
Length of stay Median/IQR \\
Respiratory complications: $\mathrm{n}(\%)$ \\
Atelectasis: $\mathrm{n}(\%)$ \\
Infectious respiratory complications: $\mathrm{n}(\%)$ \\
Acute respiratory failure: $\mathrm{n}(\%)$ \\
BPAP Day 1 ICU : $\mathrm{n}(\%)$ \\
FIO2 Day 1 ICU: Median/IQR \\
Nausea and vomiting Day $1: \mathrm{n}(\%)$ \\
Neurological complication: $\mathrm{n}(\%)$ \\
Sciatic compression: $\mathrm{n}(\%)$ \\
Brachial plexus compression: $\mathrm{n}(\%)$ \\
Returning to the operating room: $\mathrm{n}(\%)$ \\
Flap complications: $\mathrm{n}(\%)$ \\
Breast hematoma: $\mathrm{n}(\%)$ \\
Flap explantation: $\mathrm{n}(\%)$ \\
Flap necrosis : $\mathrm{n}(\%)$ \\
Venous flap complication : $\mathrm{n}(\%)$ \\
Arterial flap complication: $\mathrm{n}(\%)$ \\
\hline
\end{tabular}

\begin{tabular}{c} 
Intrathecal M+ $(\mathrm{n}=$ \\
$176)$ \\
\hline $147 / 100-205$ \\
\hline $27(15 \%)$ \\
\hline $8(5 \%)$ \\
\hline $50(28 \%)$ \\
\hline $10.9 / 9.1-13.1$ \\
\hline $14(8 \%)$ \\
\hline $6(3 \%)$ \\
\hline $5(3 \%)$ \\
\hline $2(1 \%)$ \\
$2(1 \%)$ \\
\hline $33 / 30-36$ \\
\hline $29(16 \%)$ \\
\hline $39(22 \%)$ \\
\hline $18(10 \%)$ \\
\hline $27(15 \%)$ \\
$31(18 \%)$ \\
\hline $19(11 \%)$ \\
$7(4 \%)$ \\
\hline $6(3 \%)$ \\
$19(11 \%)$ \\
\hline $13(7 \%)$ \\
$3(2 \%)$ \\
\end{tabular}

\begin{tabular}{c} 
No intrathecal M+ (n \\
$=93)$ \\
$150 / 100-205$ \\
$58(63 \%)$ \\
$5(5 \%)$ \\
$11(12 \%)$ \\
\hline $11.2 / 9.9-14.2$ \\
\hline $19(20 \%)$ \\
\hline $8(9 \%)$ \\
$6(6 \%)$ \\
$8(9 \%)$ \\
$8(9 \%)$ \\
\hline $33 / 30-36$ \\
$16(17 \%)$ \\
\hline $12(13 \%)$ \\
\hline $4(4 \%)$ \\
\hline $9(10 \%)$ \\
\hline $20(22 \%)$ \\
$9(10 \%)$ \\
$2(2 \%)$ \\
$2(2 \%)$ \\
$9(10 \%)$ \\
$8(9 \%)$ \\
$1(1 \%)$ \\
\hline
\end{tabular}

\begin{tabular}{|c|}
\hline $\mathrm{p}$ \\
\hline 0.89 \\
\hline$<0.0001$ \\
\hline 0.73 \\
\hline 0.002 \\
\hline 0.15 \\
\hline 0.003 \\
\hline 0.08 \\
\hline 0.20 \\
\hline 0.004 \\
\hline$<0.001$ \\
\hline 0.26 \\
\hline 0.86 \\
\hline 0.07 \\
\hline 0.11 \\
\hline 0.26 \\
\hline 0.44 \\
\hline 0.78 \\
\hline 0.72 \\
\hline 0.56 \\
\hline 0.84 \\
\hline 0.81 \\
\hline 0.39 \\
\hline
\end{tabular}

Morphine, which is relatively less hydrophobic than other opioids, has a longer residence time in the cerebrospinal fluid and therefore may reach rostral sites over a longer period than other opioids [26]. Consequently, there is a potential of achieving adequate and long-lasting analgesia with an intrathecal injection of morphine [27]. However, the downside of this less hydrophobic character is an increased risk of adverse effects, especially postoperative respiratory depression, [28] which remains a particular concern. This tendency was not found in our study, and no patient in the ITM group experienced respiratory depression that required re-intubation.

Preoperative administration of intrathecal morphine $(300 \mu)$ seems to be the main factor which resulted in a significant decrease in blood loss. In 2001, Gall et al. [29] studied low dose intrathecal morphine use in scoliosis correction and discovered an incidental finding: mean intraoperative blood loss was decreased with increasing doses of intrathecal morphine. Eschertzhuber et al. [30] found similar results. Our results follow the trend of those in Gall et al.'s and Eschertzhuber et al.'s in that intrathecal morphine decreased blood loss significantly.

Our study also demonstrated that decreased bleeding translated into significantly fewer blood transfusions. The number of patients transfused was significantly decreased in the ITM group (Table 2). It is well known that blood transfusions are associated with many risks, some of 
which have lifelong consequences, and as such, are best avoided especially in the oncologic population.

Several studies have shown that managing anemia in these patients with allogeneic blood transfusions can negatively impact the patients' recovery even in the absence of procedural errors [31-34]. While the cause has not been determined definitively, a physiological response known as "transfusion-related immunomodulation" or "TRIM" has been proposed as the possible mechanism [35].

Our results show that patients with hypotension were more observed in the ITM group (Table 2). Of note, intrathecal morphine-induced hypotension is also the proposed mechanism for the decrease in blood loss in the ITM group. This hypotensive effect is thought to be related to a direct sympatholytic effect of intrathecal morphine similar to that of local anesthetics [36, 37]. Morphine, when applied inside the squid axon depressed the action potential within a few minutes resulting in complete block. This block was reversible and not accompanied by depolarization of the resting membrane potential. Our study thus confirm the results of Goodarzi [38], who compared systemic analgesia with $20 \mathrm{mcg} / \mathrm{kg}$ of intrathecal morphine for postoperative pain control, reported a mean arterial pressure of $50-55 \mathrm{mmHg}$ in the intrathecal morphine group vs $65-70 \mathrm{mmHg}$ in the group that did not receive intrathecal morphine.

Intrathecal morphine produces intense analgesia for up to 24 hours with a single shot, and clinical recommendation is to choose the minimum effective dose and do not exceed $300 \mu \mathrm{g}$ to prevent the delay respiratory depression. In our study opioids consumption were significantly reduced in intra and postoperative surgery when intrathecal morphine was used.

Furthermore, both Gall et al. [29] and Eschertzhuber et al. [30] demonstrated improved post-operative analgesia when intrathecal morphine was used. The quality of analgesia after surgery affects patient outcome. Good pain control reduces the incidence of chest infections, and shortens the time spent in hospital [39].

Postoperative pain due to surgery done on the breast will lead patient to minimize chest wall movement in order to avoid the pain, with decreased functional residual capacity, and a tendency to hypoventilations. This hypothesis can explain way respiratory complications were less frequent when intrathecal morphine was combined to general anesthesia for DIEP breast reconstruction in our institution. Indeed, our study shows that general anaesthesia when combined to intrathecal morphine successfully decreased the incidence of postoperative acute respiratory failure leading to non-invasive ventilation with BPAP (Biphasic intermittent positive airway pressure) on the first postoperative day (Table 3 ).

\section{Limitations}

Limitation is mainly linked to the fact that our study is retrospective. Therefore, some information were missing in the consulted files, limiting the available variables.

Our results need replications from prospective randomized trials in order to confirm the tendencies observed with those samples. 


\section{Conclusion}

Intrathecal morphine analgesia is an effective complement to general anaesthesia and intraoperative analgesic management for DIEP flap breast reconstruction. It was associated with less blood loss and fluid administration, better postoperative pain control, and less respiratory complications than intravenous narcotic analgesia.

These findings should be confirmed in large prospective randomized trials using specific guidelines. The relatively low number of serious complications denotes an adequate preoperative screening and stresses the importance of adequate maintenance of parameters throughout the perioperative process.

\section{Competing Interests}

The authors declare no competing interests.

\section{Acknowledgement}

This project is funded by the Department of Anesthesia, Critical Care and Pain Medicine, Jules Bordet Institute. Brussels

None of the authors has a financial interest in any of the products, devices, or drugs mentioned in this manuscript.

\section{Author Contribution}

Dr. Ben Aziz Mohammed has managed literature search, figures, study and writing scientific articles for publication. Dr. Halenarova Katarina managed data collections Via computerized medical record; ORIBASE and INNOVIAN peroperative recorder reports (Anesthesia information management system to create a complete, continuous, paperless record of patient's anesthetic care). Mme Paesmans Marianne managed data analysis, statistical research and data interpretation. Linguistic revision and design of the text managed by Dr. Schroder Elisa, Dr. Kamps Victor, Dr. Khalife Maher, Dr. Sosnowski Maurice, and Dr. Urbain Frederic.

\section{References}

[1] American Cancer Society, Cancer Facts and Figures 2017. Atlanta, Ga: American Cancer Society, 2017.

[2] N. S. El Saghir, R. A. Farhat, R. N. Charara, and K. E. Khoury, "Enhancing cancer care in areas of limited resources: Our next steps," Future Oncology, vol. 10, no. 12, pp. 1953-1965, 2014.

[3] S. Mukherjee, The Emperor of All Maladies: A Biography of Cancer .Scribner Publishing House, New York, USA (2010).

[4] D. D. Atherton, A. J. Hills, P. Moradi, N. Muirhead, and S. H. Wood, "The economic viability of breast reconstruction in the UK: Comparison of a single surgeon's experience of implant; LD; TRAM and DIEP based reconstructions in 274 patients," Journal of Plastic, Reconstructive \& Aesthetic Surgery, vol. 64, no. 6, pp. 710715, 2011.

[5] H. Holmström, "The free abdominoplasty flap and its use in breast reconstruction: An experimental study and clinical case report," Journal of Plastic Surgery and Hand Surgery, vol. 13, no. 3, pp. 423-427, 1979. 
[6] R. J. Allen and P. Treece, "Deep inferior epigastric perforator flap for breast reconstruction," Annals of Plastic Surgery, vol. 32, no. 1, pp. 32-38, 1994.

[7] L. Lantieri, M. Hivelin, M. D. Benjoar et al., "Setting of a breast autologous microsurgical reconstructive surgery evolution in 20 years and review of 1138 cases," Annales de Chirurgie Plastique Esthétique, vol. 60, no. 6, pp. 484-489, 2015.

[8] I. Koshima and S. Soeda, "Inferior epigastric artery skin flaps without rectus abdominis muscle," British Journal of Plastic Surgery, vol. 42, no. 6, pp. 645-648, 1989.

[9] R. J. Allen and P. Treece, "Deep inferior epigastric perforator flap for breast reconstruction," Annals of Plastic Surgery, vol. 32, no. 1, pp. 32-38, 1994.

[10] M. Hamdi, E. M. Weiler-Mithoff, and M. H. C. Webster, "Deep inferior epigastric perforator flap in breast reconstruction: experience with the first 50 flaps," Plastic and Reconstructive Surgery, vol. 103, no. 1, pp. 8695, 1999.

[11] M. Hamdi, P. Blondeel, K. Van Landuyt, T. Tondu, and S. Monstrey, "Bilateral autogenous breast reconstruction using perforator free flaps: A single center's experience," Plastic and Reconstructive Surgery, vol. 114, no. 1, pp. 83-89, 2004.

[12] B. Tsoi, N. I. Ziolkowski, A. Thoma, K. Campbell, D. O'Reilly, and R. Goeree, "Safety of tissue expander/implant versus autologous abdominal tissue breast reconstruction in postmastectomy breast cancer patients: A systematic review and meta-analysis," Plastic and Reconstructive Surgery, vol. 133, no. 2, pp. 234249, 2014.

[13] F. Bonnet and E. Marret, "Influence of anaesthetic and analgesic techniques on outcome after surgery," British Journal of Anaesthesia, vol. 95, no. 1, pp. 52-58, 2005.

[14] T. Zhong, K. W. Wong, H. Cheng et al., "Transversus abdominis plane (TAP) catheters inserted under direct vision in the donor site following free DIEP and MS-TRAM breast reconstruction: A prospective cohort study of 45 patients," Journal of Plastic, Reconstructive \& Aesthetic Surgery, vol. 66, no. 3, pp. 329-336, 2013.

[15] N. V. Tran, E. W. Buchel, and P. A. Convery, "Microvascular complications of DIEP flaps," Plastic and Reconstructive Surgery, vol. 119, no. 5, pp. 1397-1405, 2007.

[16] Lim J Yap YL, The deep inferior epigastric perforator flap for breast reconstruction: is this the ideal flap for Asian women? Ann Acad Med Singapore 2010; 39: 680-686.

[17] M. F. Massey, A. J. Spiegel, J. L. Levine et al., "Perforator flaps: Recent experience, current trends, and future directions based on 3974 microsurgical breast reconstructions," Plastic and Reconstructive Surgery, vol. 124, no. 3, pp. 737-751, 2009.

[18] D. Eran, H. Bar-Meir a Janet, and E. a Philip, "Postoperative Pain Management in DIEP Flap Breast Reconstruction: Identification of Patients With Poor Pain Control. Ann R Coll Surg Engl," in MD, vol. 94, pp. 552-558, 552-558, 94(8, Nov 2012.

[19] F. Bonnet and E. Marret, "Influence of anaesthetic and analgesic techniques on outcome after surgery," British Journal of Anaesthesia, vol. 95, no. 1, pp. 52-58, 2005.

[20] U. M. Stamer, S. Grond, and C. Maier, "Responders and non-responders to post-operative pain treatment: The loading dose predicts analgesic needs," European Journal of Anaesthesiology, vol. 16, no. 2, pp. 103-110, 1999.

[21] R. H. Enting, W. H. Oldenmenger, C. C. D. Van Der Rijt et al., "A prospective study evaluating the response of patients with unrelieved cancer pain to parenteral opioids," Cancer, vol. 94, no. 11, pp. 3049-3056, 2002.

[22] J. K. Wang, L. A. Nauss, and J. E. Thomas, "Pain relief by intrathecally applied morphine in man," Anesthesiology, vol. 50, no. 2, pp. 149-151, 1979.

[23] A. Borgeat, G. Ekatodramis, and C. A. Schenker, "Postoperative nausea and vomiting in regional anesthesia: A review," Anesthesiology, vol. 98, no. 2, pp. 530-547, 2003.

[24] S. S. Liu, B. M. Block, and C. L. Wu, "Effects of perioperative central neuraxial analgesia on outcome after coronary artery bypass surgery: a meta-analysis," Anesthesiology, vol. 101, no. 1, pp. 153-161, 2004.

[25] J. P. Rathmell, T. R. Lair, and B. Nauman, "The Role of Intrathecal Drugs in the Treatment of Acute Pain," Anesthesia \& Analgesia, vol. 101, no. Supplement, pp. S30-S43, 2005.

[26] W. C. Ummenhofer, R. H. Arends, D. D. Shen, and C. M. Bernards, "Comparative spinal distribution and clearance kinetics of intrathecally administered morphine, fentanyl, alfentanil, and sufentanil," Anesthesiology, vol. 92, no. 3, pp. 739-753, 2000.

[27] S. S. Liu, B. M. Block, and C. L. Wu, "Effects of perioperative central neuraxial analgesia on outcome after coronary artery bypass surgery: a meta-analysis," Anesthesiology, vol. 101, no. 1, pp. 153-161, 2004.

[28] C. J. Law and E. J. Visser, "Unconsciousness and severe respiratory depression following intrathecal morphine analgesia for lumbar spinal surgery," Acute Pain, vol. 9, no. 3, pp. 163-167, 2007.

[29] O. Gall, J.-V. Aublneau, J. Berniére, L. Desjeux, and I. Murat, "Analgesic effect of low-dose intrathecal morphine after spinal fusion in children," Anesthesiology, vol. 94, no. 3, pp. 447-452, 2001.

[30] S. Eschertzhuber, M. Hohlrieder, C. Keller, E. Oswald, G. Kuehbacher, and P. Innerhofer, "Comparison of highand low-dose intrathecal morphine for spinal fusion in children," British Journal of Anaesthesia, vol. 100, no. 4, pp. 538-543, 2008. 
[31] A. Amato and M. Pescatori, "Perioperative blood transfusions for the recurrence of colorectal cancer," Cochrane Database of Systematic Reviews, Article ID CD005033, 2006.

[32] E. C. Vamvakas and M. A. Blajchman, "Transfusion-related mortality: the ongoing risks of allogeneic blood transfusion and the available strategies for their prevention," Blood, vol. 113, no. 15, pp. 3406-3417, 2009.

[33] C. Perisanidis, M. Dettke, N. Papadogeorgakis et al., "Transfusion of allogenic leukocyte-depleted packed red blood cells is associated with postoperative morbidity in patients undergoing oral and oropharyngeal cancer surgery," Oral Oncology, vol. 48, no. 4, pp. 372-378, 2012.

[34] C. Miki, E. Ojima, Y. Inoue, Y. Mohri, and M. Kusunoki, "Perioperative allogeneic blood transfusion, the related cytokine response and long-term survival after potentially curative resection of colorectal cancer," Clinical Oncology, vol. 18, no. 1, pp. 60-66, 2006.

[35] E. C. Vamvakas and M. A. Blajchman, "Transfusion-related immunomodulation (TRIM): an update,” Blood Reviews, vol. 21, no. 6, pp. 327-348, 2007.

[36] D. T. Frazier, K. Murayama, N. J. Abbott, and T. Narahashi, "Effects of Morphine on Internally Perfused Squid Giant Axons," Proceedings of the Society for Experimental Biology and Medicine, vol. 139, no. 2, pp. 434-438, 1972.

[37] P. Brodin and L. A. Skoglund, "Dose-response inhibition of rat compound nerve action potential by dextropropoxyphene and codeine compared to morphine and cocaine in vitro," General Pharmacology: The Vascular System, vol. 21, no. 4, pp. 551-553, 1990.

[38] M. Goodarzi, "The advantages of intrathecal opioids for spinal fusion in children," Pediatric Anesthesia, vol. 8, no. 2, pp. 131-134, 1998.

[39] J. C. Crews, “Epidural opioid analgesia,” Critical Care Clinics, vol. 6, no. 2, pp. 315-342, 1990. 